\title{
The combination of chronic kidney disease and increased arterial stiffness is a predictor for stroke and cardiovascular disease in hypertensive patients
}

\author{
Mitsuru Ohishi, Yuji Tatara, Norihisa Ito, Yasushi Takeya, Miyuki Onishi, Yoshihiro Maekawa, \\ Nozomi Kato, Kei Kamide and Hiromi Rakugi
}

To clarify the clinical utility of pulse wave velocity (PWV) and chronic kidney disease (CKD) in hypertension, we analyzed the prognostic impact of PWV and CKD on cerebrocardiovascular disease in hypertensive patients. This study consisted of 531 patients with essential hypertension (male/female $=292 / 239$, mean age $=61.7 \pm 12.3$, mean follow-up $=7.0 \pm 3.0$ years) and was performed between January 1998 and June 2004 . We used questionnaires to assess stroke $(n=57)$, cardiovascular diseases (CVDs; myocardial infarction, angina and congestive heart failure; $n=44)$ and death $(n=53)$ as primary end points. At baseline, we evaluated the carotid-femoral PWV $\left(9.1 \pm 1.8 \mathrm{~m} \mathrm{~s}^{-1}\right)$, the glomerular filtration rate and urinary protein excretions. We divided these subjects into those in the highest quartile of PWV and other subjects and into CKD $(n=149)$ and non-CKD $(n=458)$. We evaluated the prognostic influences of PWV and CKD with Kaplan-Meier analysis and Cox's proportional hazard model. PWV in CKD $\left(9.6 \pm 1.9 \mathrm{~m} \mathrm{~s}^{-1}\right)$ was higher than in non-CKD $\left(8.8 \pm 1.6 \mathrm{~m} \mathrm{~s}^{-1} ; P<0.0001\right)$, and creatinine was slightly decreased in the highest PWV group $\left(1.09 \pm 0.35 \mathrm{mg} \mathrm{dl}^{-1}, P<0.0001\right)$. On the basis of Kaplan-Meier analysis, the highest PWV group (PWV $\left.>10.1 \mathrm{~m} \mathrm{~s}^{-1} ; P=0.0003\right)$ and the CKD group $(P=0.0005)$ showed significantly higher proportions of stroke and CVD events. In addition, the highest PWV group showed the highest percentage of stroke $(P=0.0007)$, and the CKD group showed the highest proportion of CVD $(P<00001)$. High PWV and CKD were independent predictors for stroke and CVD $(P=0.0332)$ by Cox's proportional hazard model. These data suggest that increased aortic stiffness and CKD may be predictors for stroke and cardiovascular events in hypertensive patients.

Hypertension Research (2011) 34, 1209-1215; doi:10.1038/hr.2011.117; published online 4 August 2011

Keywords: arterial stiffness; chronic kidney disease; cohort study

\section{INTRODUCTION}

Recently, chronic kidney disease (CKD) has been examined not only in relation to renal failure but also as a risk factor for cardiovascular diseases (CVD). ${ }^{1}$ In patients with $\mathrm{CKD}$, cardiovascular death is far more common than dialysis at all stages. ${ }^{2}$ A prominent epidemiological study, called the Hisayama study, suggested that CKD is one of the risk factors for ischemic heart disease but not stroke. ${ }^{3}$ Large epidemiological datasets suggest that even mild renal disease, as assessed by the estimated glomerular filtration rate (GFR), is a major risk factor for cardiovascular events after myocardial infarction. ${ }^{4} \mathrm{CKD}$ is also associated with arterial stiffness, as evaluated by pulse wave velocity (PWV), and intima-media thickening of the carotid artery. ${ }^{5}$ Decreased estimated GFR is associated with arterial stiffness in a Japanese population, and high PWV is a risk factor for cardiovascular mortality in patients with end-stage renal damage. ${ }^{6}$

High PWV is also a major risk factor for stroke and coronary artery disease in hypertensive patients. ${ }^{7}$ We reported in a cohort study, called the Non-invasive Atherosclerotic Evaluation in Hypertension study, ${ }^{8}$ that having a carotid-femoral PWV in the highest quartile is an independent risk factor for stroke and CVD in hypertensive patients. Both arterial stiffness and CKD are important risk factors for stroke and CVD; however, the ability of the relationship among these risk factors to predict stroke and cardiovascular disease in hypertensive patients has not been reported. Therefore, we analyzed the predictive value of the combination of PWV and CKD for stroke and CVD in hypertensive patients as a part of the Non-invasive Atherosclerotic Evaluation in Hypertension study.

\section{METHODS}

Study population and study design

This cohort study was designed as part of the Non-invasive Atherosclerotic Evaluation in Hypertension study. ${ }^{8}$ The Non-invasive Atherosclerotic Evaluation in Hypertension study analyzed outpatients who were randomly recruited and had been diagnosed as having essential hypertension between January 1998 and June 2004. We extended the follow-up period for every patient to September 2010, as described in the next paragraph. This part of the study 
Table 1 Baseline clinical characteristics

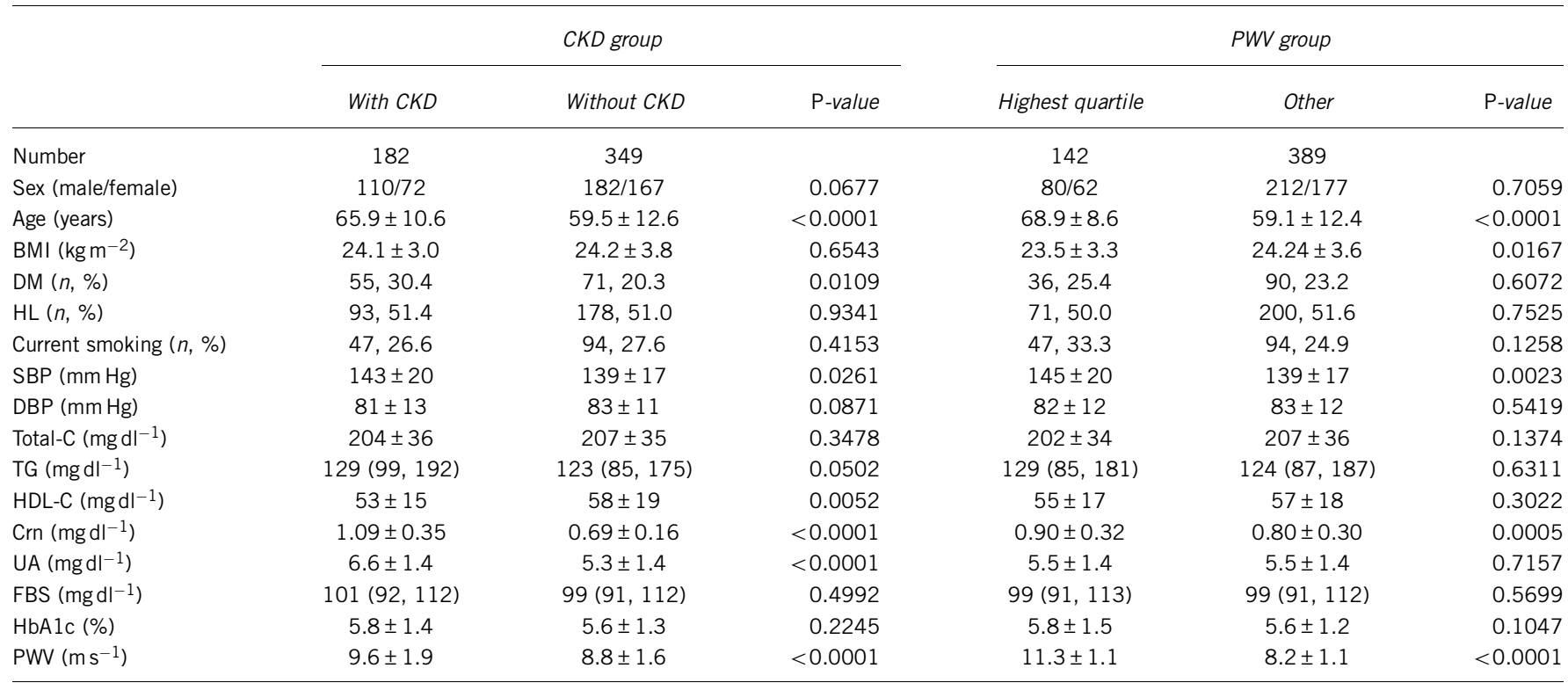

Abbreviations: BMI, body mass index; Crn, creatinine; CKD, chronic kidney disease; DBP, diastolic blood pressure; DM, diabetes mellitus; FBS, fasting blood sugar; HbAlc, hemoglobin A1c; HDL-C, high-density lipoprotein-cholesterol; HL, total cholesterol $>220 \mathrm{mg} \mathrm{dl}^{-1}$ and/or triglyceride $>150 \mathrm{mg} \mathrm{dl}^{-1}$; PWV, pulse wave velocity; SBP, systolic blood pressure; TG, triglyceride, total-C; total cholesterol; UA, uric acid.

Data was expressed as mean \pm s.d. except for TG and FBS. Data for TG and FBS was expressed as median (1st quartile, 3rd quartile). P-value for TG and FBS was calculated after log transformation.

investigated 531 hypertensive outpatients, as shown in Table 1. We excluded patients with the presence or history of arteriosclerosis obliterans and patients with an ankle-brachial index of less than 0.9. The study protocol was approved by the ethics committee of Osaka University Hospital, and written informed consent was obtained from all participants. At baseline, a total of 246 patients were not being treated with anti-hypertensive drugs, and 285 patients were being treated with one or more anti-hypertensive drugs: 196 patients with a calcium antagonist, 91 patients with an angiotensin II receptor blocker, 87 patients with an angiotensin-converting enzyme inhibitor, 58 patients with a $\beta$ blocker, 37 patients with a diuretic and 16 patients with an $\alpha$ blocker. An additional 107 patients were also being treated with a statin. At baseline, 43 patients had a history of CVD and 11 patients had a history of stroke; however, patients with obvious neurological disturbances were excluded.

\section{Follow-up evaluation}

Clinical follow-up was conducted by clinical visits, mailed questionnaires and telephone contact every September starting in 2003. The questionnaire included questions regarding the hypertensive complications described below and cause of death. We also confirmed responses in detail by comparing them against the patients' medical histories. The primary endpoint of this study was new onset of stroke or CVD, such as a new onset of angina pectoris, myocardial infarction or heart failure. The follow-up duration was considered to encompass the interval from the initial evaluation to the time of event onset or September 2010. The average follow-up period was $7.0 \pm 3.0$ years.

\section{Determination and classification of CKD}

CKD was defined as either kidney damage or GFR $<60 \mathrm{ml} \mathrm{min}^{-1}$ per $1.73 \mathrm{~m}^{2}$ according to the Kidney Disease Outcomes Quality Initiative Clinical Practice Guidelines. ${ }^{9}$ Increased urinary albumin/creatinine ratio $>30 \mathrm{mgg}^{-1}$ and/or urinary protein/creatinine ratio $>200 \mathrm{mg} \mathrm{g}^{-1}$ were used as evidence of kidney damage. The GFR was calculated using the abbreviated version of the Modification of Diet in Renal Disease study equation: GFR $\left(\mathrm{ml} \mathrm{min}^{-1}\right.$ per $\left.1.73 \mathrm{~m}^{2}\right)=194 \times \mathrm{SCr}^{-1.094} \times$ age $^{-0.287}$ (if female $\times 0.739$ ), where $\mathrm{SCr}$ is the serum creatinine concentration in $\mathrm{mg} \mathrm{dl}^{-1} .10$

\section{Determination of PWV}

We evaluated carotid-femoral PWV as a representative PWV to evaluate arterial stiffness with the patient in a supine position after $20 \mathrm{~min}$ of rest. We determined PWV using an FCP-4731 device (Fukuda Denshi Co., Tokyo, Japan), which allowed online pulse wave recording and automatic calculation. The pulse waveforms of the right carotid and femoral arteries were recorded non-invasively using a TY-306-Fukuda pressure-sensitive transducer (Fukuda Denshi Co.), and heart sounds were recorded at the same time. A preprocessor automatically analyzed the gain in each waveform and adjusted it to ensure equality of the two signals. This procedure has been published in detail elsewhere. ${ }^{11}$ To analyze the prognosis of the study population, we classified the subjects into two groups: highest PWV quartile and 'other.'

\section{Statistical analysis}

The values summarized in Table 1 are presented as the mean \pm s.d., except for triglyceride and fasting blood sugar; these parameters have non-normal distributions and are shown as median \pm 1 st quartile and 3rd quartile. In all analyses, triglyceride and fasting blood sugar levels are presented after log transformation in Tables 1 and 2. Statistical analysis was performed using analysis of variance and then a post hoc test adjusted using the Bonferroni/ Dunn correction. An event-free survival curve was estimated via the KaplanMeier method and the log-rank test was used to compare the differences in event-free rates. To examine the statistical significance between each quartile, we used a non-adjusted Cox proportional hazards model to evaluate relative risk (RR). Baseline clinical variables for these patients were analyzed with the Cox proportional hazards model confounded with common risk factors such as gender, age, presence of diabetes, systolic blood pressure (SBP), and levels of high-density lipoprotein cholesterol and uric acid. Analyses were performed with commercially available software (JMP version 8, SAS Ins., Cary, NC, USA). Findings of $P<0.05$ were considered significant.

\section{RESULTS}

\section{Patient characteristics}

Baseline clinical characteristics are summarized in Table 1. A total of $34.3 \%$ of patients ( $n=182$ of 531 ) were diagnosed as having CKD. Age, percentage with diabetes, SBP, serum creatinine level, serum uric acid 
Table 2 Cox proportional hazard model

\begin{tabular}{|c|c|c|c|c|c|c|}
\hline & \multicolumn{2}{|c|}{ Stroke and CVD } & \multicolumn{2}{|c|}{ Stroke } & \multicolumn{2}{|c|}{$C V D$} \\
\hline & $R R$ & P-value & $R R$ & P-value & $R R$ & P-value \\
\hline \multicolumn{7}{|l|}{ PWV } \\
\hline Low & 1 & & 1 & & 1 & \\
\hline High & 2.104 & 0.0007 & 2.410 & 0.0014 & 1.706 & 0.0980 \\
\hline With & 2.027 & 0.0008 & 1.446 & 0.1744 & 3.196 & 0.0001 \\
\hline \multicolumn{7}{|l|}{ CKD } \\
\hline Without CKD & 1 & 0.0098 & 1 & 0.1775 & 1 & 0.0013 \\
\hline \multicolumn{7}{|l|}{ Severity } \\
\hline Stage 1 and 2 & 2.158 & 0.0843 & 1.707 & 0.3464 & 3.369 & 0.0552 \\
\hline Stage 3 & 1.984 & 0.0025 & 1.511 & 0.1535 & 2.922 & 0.0013 \\
\hline High PWV without CKD & 1.379 & 0.3495 & 2.319 & 0.0270 & 4.201 & 0.0836 \\
\hline High PWV with CKD & 3.712 & $<0.0001$ & 2.957 & 0.0039 & 4.437 & $<0.0001$ \\
\hline \multicolumn{7}{|l|}{ Adjusted model 1} \\
\hline \multicolumn{7}{|l|}{ PWV } \\
\hline Low & 1 & & 1 & & 1 & \\
\hline High & 1.600 & 0.0519 & 1.887 & 0.0362 & 1.074 & 0.8392 \\
\hline \multicolumn{7}{|l|}{$C K D$} \\
\hline Without & 1 & & 1 & & 1 & \\
\hline With & 1.332 & 0.2215 & 1.041 & 0.8945 & 1.790 & 0.0923 \\
\hline \multicolumn{7}{|l|}{ CKD } \\
\hline Without CKD & 1 & 0.7149 & 1 & 0.2975 & 1 & 0.3938 \\
\hline High PWV with CKD & 2.150 & 0.0332 & 1.911 & 0.1108 & 1.872 & 0.2045 \\
\hline \multicolumn{7}{|l|}{ Adjusted model 2} \\
\hline \multicolumn{7}{|l|}{ PWV } \\
\hline Low & 1 & & 1 & & 1 & \\
\hline High & 1.577 & 0.0746 & 1.905 & 0.0478 & 1.037 & 0.9218 \\
\hline \multicolumn{7}{|l|}{ CKD } \\
\hline Without & 1 & & 1 & & 1 & \\
\hline With & 1.318 & 0.2438 & 0.981 & 0.9516 & 1.910 & 0.0747 \\
\hline CKD & & & & & & \\
\hline Without CKD & 1 & 0.5744 & 1 & 0.4931 & 1 & 0.2962 \\
\hline Severity & & & & & & \\
\hline Stage 1 and 2 & 1.651 & 0.3518 & 1.203 & 0.6567 & 2.734 & 0.1875 \\
\hline Stage 3 & 1.305 & 0.2502 & 0.998 & 0.8294 & 1.843 & 0.1206 \\
\hline Stage 4 and 5 & 0.821 & 0.7180 & 0.001 & 0.1571 & 1.520 & 0.2120 \\
\hline PWV and CKD & & & & & & \\
\hline Low PWV without CKD & 1 & 0.0667 & 1 & 0.2524 & 1 & 0.0220 \\
\hline Low PWV with CKD & 0.902 & 0.9093 & 0.838 & 0.7301 & 1.060 & 06469 \\
\hline High PWV without CKD & 0.994 & 0.9548 & 1.756 & 0.1394 & 0.176 & 0.0416 \\
\hline High PWV with CKD & 2.087 & 0.0376 & 1.810 & 0.1055 & 1.942 & 0.2596 \\
\hline
\end{tabular}

Abbreviations: CKD, chronic kidney disease; CVD, cardiovascular disease; PWV, pulse wave velocity; RR, relative risk.

Confounding factor for model 1: age, presence of diabetes, systolic blood pressure, uric acid and body mass index.

Confounding factor for model 2: age, gender, presence of diabetes, systolic blood pressure, uric acid, body mass index, high-density lipoprotein-cholesterol, anti-hypertensive medication, presence of current smoking, anti-lipidemic medication and triglyceride after log transformation. 
level and PWV were higher in patients with CKD than in patients without CKD, and high-density lipoprotein cholesterol was lower in patients with CKD than in patients without CKD. The characteristics of patients with the highest PWV vs. other patients are shown in the right column of Table 1. Age, SBP, serum creatinine level and PWV in patients with the highest quartile PWV were higher than in other patients, and body mass index in patients with the highest PWV was lower than in other patients. Moreover, the PWV in patients with CKD $\left(9.85 \pm 2.06 \mathrm{~m} \mathrm{~s}^{-1}\right)$ was significantly higher than the PWV in patients without CKD $\left(8.93 \pm 1.72 \mathrm{~m} \mathrm{~s}^{-1}\right)$, but this difference was observed only in male patients $(P<0.0001$; female patients: $\left.9.35 \pm 2.03 \mathrm{~m} \mathrm{~s}^{-1} ; 9.02 \pm 1.66 \mathrm{~m} \mathrm{~s}^{-1} ; P=0.1896\right)$.
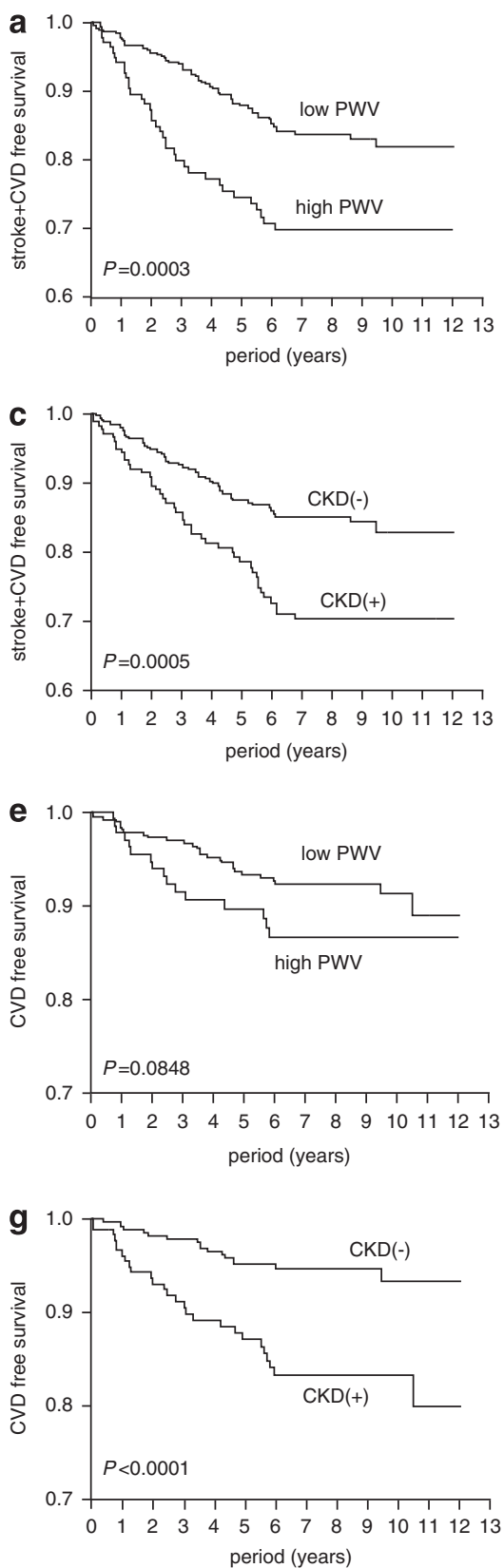

Kaplan-Meier analysis classified by CKD or PWV

By the time of the follow-up survey, 93 of the 531 patients $(17.5 \%)$ had suffered from stroke or CVD, 57 (10.7\%) had suffered from stroke only, $44(8.3 \%)$ had suffered from CVD only and 53 (10.0\%) patients had died. Figure 1 shows the results of the Kaplan-Meier analysis for the occurrence of stroke or CVD (a and $\mathbf{c}$ ) and for total mortality (b and $\mathbf{d}$ ), classified by CKD (c and $\mathbf{d}$ ) and PWV (a and $\mathbf{b}$ ). The eventfree survival proportions of the highest quartile of PWV $\left(>10.1 \mathrm{~m} \mathrm{~s}^{-1} ; P=0.0003\right)$ and for patients with CKD $(P=0.0005)$ with regard to the occurrence of stroke or CVD were significantly lower by the log-rank test. The event-free survival proportions of the patients with the highest quartile of PWV $(P<0.0001)$ or the presence
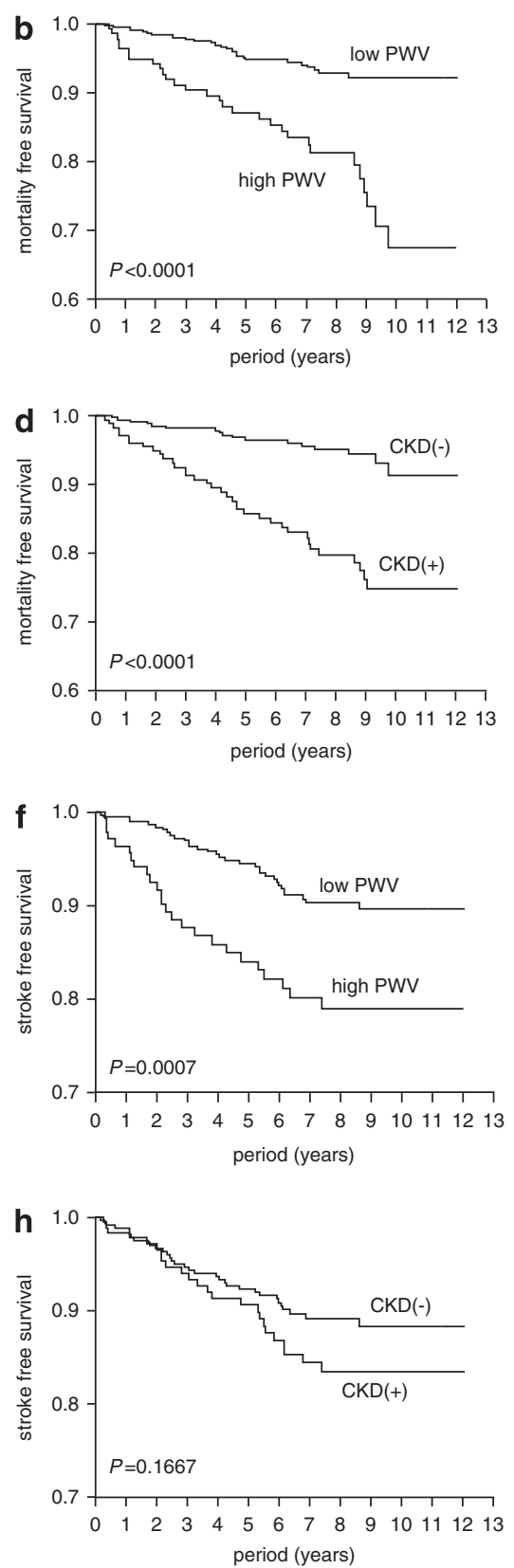

Figure 1 Kaplan-Meier analysis. (a) Stroke or cardiovascular disease (CVD) classified by pulse wave velocity (PWV) quartile. (b) Total mortality classified by PWV quartile. (c) Stroke or CVD classified by presence or absence of chronic kidney disease (CKD). (d) Total mortality classified by presence or absence of CKD. (e) CVD classified by PWV quartile. (f) Stroke classified by PWV quartile. (g) CVD classified by presence or absence of CKD. (h) Stroke classified by presence or absence of CKD. -------, other PWV quartiles or without CKD; -, highest PWV quartile or with CKD. 
of CKD $(P<0.0001)$ with regard to total mortality were also significantly lower by the log-rank test. The event-free survival proportion of those in the highest quartile of PWV with regard to the occurrence of stroke was significantly lower than in the other patients (Figure 1f; $P=0.0007$ ); however, of the occurrence of CVD showed no differences between the PWV-related groups (Figure 1e). The event-free survival proportions of patients with CKD with regard to the occurrence of CVD was significantly lower than in patients without CKD (Figure 1g; $P<0.0001$ ); however, the relationship with stroke showed no difference in these two groups of patients (Figure 1h).

\section{Severity of CKD and combined analysis of PWV and CKD}

We also analyzed influences of the severity of CKD classified by Kidney Disease: Improving Global Outcomes (1 and 2, 3, and 4 and 5) on the occurrence of stroke and CVD, as shown in Figure 2a. The severity of CKD classified by Kidney Disease: Improving Global Outcomes had a statistical influence $(P=0.0069$ by log-rank test) on the occurrence of stroke or CVD. The Kaplan-Meier curve revealed that patients without CKD had a lower risk of occurrence of stroke or CVD; however, each class of CKD had a similar risk of stroke or CVD.

A Kaplan-Meier curve of the combined event-free survival in relation to PWV and CKD is shown in Figure 2b. More than $40 \%$ of patients with CKD and the highest quartile of PWV had a stroke or CVD event in a 6-year period, whereas fewer than $15 \%$ patients without CKD and in other PWV quartiles had a stroke or CVD event in a 10-year period. Combined analysis of CKD and PWV appears to have high prognostic value for stroke or CVD events in patients with essential hypertension.

\section{Cox proportional hazards model}

We performed Cox proportional hazards modeling to examine the highest quartile of PWV, the presence of $\mathrm{CKD}, \mathrm{CKD}$ severity and the combination of PWV and CKD for prediction of stroke, CVD and stroke or CVD (Table 2). Using the non-adjusted model, the highest quartile value of $\mathrm{PWV}(\mathrm{RR}=2.104 ; P=0.0007)$, the presence of $\mathrm{CKD}$ $(\mathrm{RR}=2.027 ; P=0.0008)$, the severity of $\mathrm{CKD}$ (stage $3 ; \mathrm{RR}=1.984$; $P=0.0025$ vs. without $\mathrm{CKD}$ ) and the combination of $\mathrm{CKD}$ and $\mathrm{PWV}$ (highest PWV with $\mathrm{CKD}$; $\mathrm{RR}=3.712 ; P<0.0001$ vs. low $\mathrm{PWV}$ without $\mathrm{CKD}$ ) were risk factors for stroke or CVD. Only the highest quartile value of $\mathrm{PWV}(\mathrm{RR}=2.410 ; P=0.0014)$ and combination of $\mathrm{CKD}$ and PWV (highest PWV without $\mathrm{CKD}$; $\mathrm{RR}=2.319 ; P=0.0270$, high $\mathrm{PWV}$ with $\mathrm{CKD} ; \mathrm{RR}=2.957 ; P=0.0039$ vs. low $\mathrm{PWV}$ without $\mathrm{CKD}$ ) were risk factors for stroke, and the presence of $\mathrm{CKD}(\mathrm{RR}=3.196$;
$P=0.0001$ ), the severity of $\mathrm{CKD}$ (stage $3 ; \mathrm{RR}=2.922 ; P=0.0013$, stage 4 and $5 ; \mathrm{RR}=6.052 ; P=0.0197$ vs. without $\mathrm{CKD}$ ), and combination of $\mathrm{CKD}$ and $\mathrm{PWV}$ (highest $\mathrm{PWV}$ with $\mathrm{CKD}$; $\mathrm{RR}=4.437 ; P<0.0001$ vs. low PWV without CKD) were risk factors for CVD.

We also performed Cox proportional hazards model with confounding factors, which showed significant differences in the CKD groups and PWV groups, shown in Table 1, with regard to factors such as age, presence of diabetes, SBP, serum uric acid and body mass index (adjusted model 1 in Table 2). Having PWV values in the highest quartile $(\mathrm{RR}=1.600 ; P=0.0519)$ resulted in a higher but not statistically significant risk for stroke and CVD, and combination of CKD and PWV (highest PWV with $\mathrm{CKD} ; \mathrm{RR}=2.150 ; P=0.0332$ vs. low PWV without CKD) emerged as a risk factor for stroke or CVD. Having only $\mathrm{PWV}$ values in the highest quartile $(\mathrm{RR}=1.887$; $P=0.0362)$ emerged as a risk factor of stroke, and the presence of $\mathrm{CKD}(\mathrm{RR}=1.790 ; P=0.0923)$ showed a higher but not statistically significant risk for CVD. We also performed Cox proportional hazards modeling with common risk factors, such as age, sex, presence of diabetes, being a current smoker, body mass index, SBP, triglyceride, high-density lipoprotein cholesterol, serum uric acid, the presence of antihypertensive medication and anti-lipidemic medication (adjusted model 2 in Table 2). Having PWV values in the highest quartile ( $\mathrm{RR}=1.577 ; P=0.0746)$ was associated with a higher but not statistically significant risk for stroke or CVD, and the combination of CKD and PWV (highest PWV with $\mathrm{CKD} ; \mathrm{RR}=2.087 ; P=0.0376$ vs. low PWV without CKD) emerged as a risk factor for stroke or CVD. Having only $\mathrm{PWV}$ values in the highest quartile $(\mathrm{RR}=1.905$; $P=0.0478)$ emerged as a risk factor for stroke, and the presence of $\mathrm{CKD}(\mathrm{RR}=1.910 ; P=0.0747)$ showed an increased but not statistically significant risk factor for CVD.

\section{DISCUSSION}

In this cohort study, we showed that combined measurement of high levels of PWV and the presence of CKD may have a strong predictive value for stroke and CVD in hypertensive patients. Moreover, we demonstrated that having a high PWV value may be a risk factor for stroke and that the presence of CKD may be a risk factor for CVD in hypertensive patients.

There have been several reports that have revealed an association between CKD and arterial stiffness in cross-sectional studies. ${ }^{12-13}$ These reports have suggested that arterial stiffness measured by PWV is associated with the presence of CKD or estimated GFR, which is similar to the results observed as baseline characteristics in this study. Although there have been many reports on the prognostic
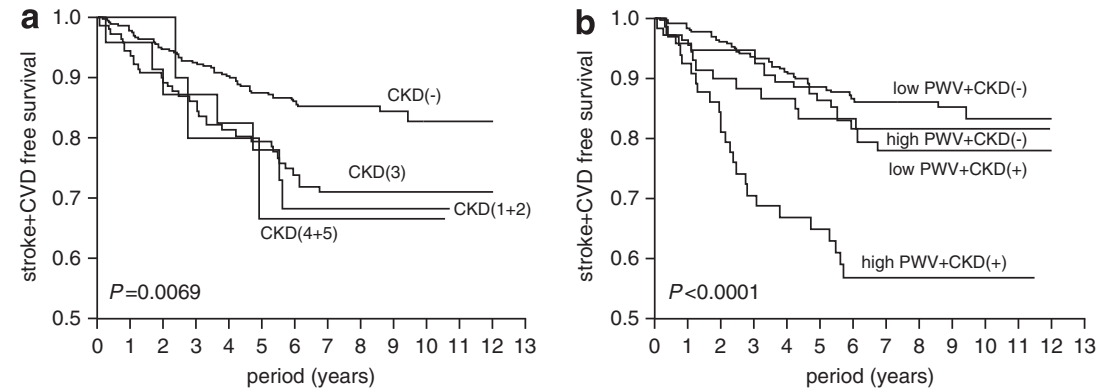

Figure 2 (a) Kaplan-Meier analysis for stroke or cardiovascular disease (CVD) due to chronic kidney disease (CKD) severity classified by Kidney Disease: Improving Global Outcome. -..--, without CKD; -----, stages 1 and $2 ;. . . . . . . . . .$. , stage 3 ; _- , stages 4 and 5 . (b) Kaplan-Meier analysis for stroke

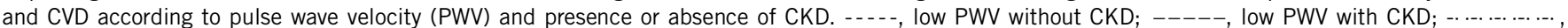
high PWV without CKD; — high PWV with CKD. 
impacts of CKD, sub-analysis of the Hisayama study suggested that the presence of CKD is an independent risk factor for CVD and coronary heart disease, but not stroke, in a general Japanese population. ${ }^{3}$ In this study, presence of CKD was also a risk for CVD, but not stroke, in outpatients with essential hypertension. The Framingham Heart Study revealed ${ }^{14}$ that having aortic PWV values in the highest quartile is a risk factor for CVD or stroke by KaplanMeier analysis. The Rotterdam Study ${ }^{15}$ also revealed that having aortic PWV values in the highest tertile is a risk factor for CVD and stroke using a Cox's proportional hazards model. In this study, having PWV values in the highest quartile was a risk factor for CVD and stroke in outpatients with essential hypertension. A longitudinal study by Laurent et al. ${ }^{16}$ suggested that increased aortic stiffness as measured by higher $\mathrm{PWV}$ is a risk factor for coronary heart disease in hypertensive patients.

Why are PWV values in the highest quartile a risk for stroke whereas the presence of CKD is a risk for CVD? The results from the subanalysis of the Hisayama study are similar to our results in a general Japanese population. ${ }^{3}$ Stroke is influenced to a greater extent by the duration and severity of pressure overload, that is, the history and severity of hypertension, whereas CVD, especially acute coronary syndrome, is based on coronary atherosclerosis. Recently, inflammatory responses ${ }^{17}$ and oxidative stress ${ }^{18}$ have been considered to be major mechanisms involved in the development of atherosclerosis. Renal dysfunction is also influenced by inflammatory responses and increased reactive oxygen species. ${ }^{19-20}$ The age-related increase in arterial stiffness is augmented in phases according to the severity of hypertension, ${ }^{21}$ and $\mathrm{PWV}$ is an independent predictor of the longitudinal increase in SBP and of hypertensive incidents in the Baltimore Longitudinal Study of Aging. ${ }^{22}$ Together with these findings, the presence of CKD may be considered a risk factor for CVD via increased inflammatory responses and oxidative stress, and higher PWV may be thought of as a risk factor for stroke based on the history and severity of hypertension.

We propose a more sensitive evaluation, combining presence of $\mathrm{CKD}$ with being in the highest quartile of PWV to predict the incidence of stroke and CVD in hypertensive patients. More than $40 \%$ of hypertensive patients with CKD and high PWV $\left(>10.1 \mathrm{~m} \mathrm{~s}^{-1}\right)$ had strokes or CVD events during a 6-year period as assessed by Kaplan-Meier analysis. In our Cox's proportional hazards model, the presence of CKD and high PWV was an independent risk $(R R=2.15)$ after adjustment of baseline characteristics. Although a further largescale clinical survey will be required, the presence of $\mathrm{CKD}$ and increased arterial stiffness may have good predictive value in the management of patients with essential hypertension.

\section{Study limitations}

The present study has several limitations. First, this cohort study was hospital-based and not a multicenter study; to avoid study bias and confirm the present results, larger cohort and multicenter trials are needed. Second, patients who enrolled in this study were receiving many types of antihypertensive treatment, and some patients were also receiving statin therapy. Recent reports indicate that treatments such as angiotensin-converting enzyme inhibitors, ${ }^{11}$ angiotensin II receptor blocker, ${ }^{23}$ or statins ${ }^{24}$ improve arterial stiffness. As a result, taking these medications may contribute to better effects on survival. Because these medications would affect the prognosis of hypertensive patients, further studies based on the type of medications are needed. Third, patients with PWV values in the highest quartile and CKD were older and had higher systolic blood pressure in the present study. These determinants may accelerate the progression of aortic stiffness during the follow-up period, resulting in a worse outcome. However, this limitation does not influence the present conclusions, as PWV values in the highest quartile and CKD were accepted as independent risk factors by the Cox proportional hazards model.

\section{ACKNOWLEDGEMENTS}

This study was funded by a Grant-in-Aid (no. 16590684) from the Ministry of Education, Science, and Culture and by a grant from Japan Arteriosclerosis Prevention Fund. We thank Ms Aki Takeda for her excellent administrative assistance.

1 Go AS, Chertow GM, Fan D, McCulloch CE, Hsu CY. Chronic kidney disease and the risks of death, cardiovascular events, and hospitalization. N Engl J Med 2004; 351: 1296-1305.

2 Keith DS, Nichols GA, Gullion CM, Brown JB, Smith DH. Longitudinal follow-up and outcomes among a population with chronic kidney disease in a large managed care organization. Arch Intern Med 2004; 164: 659-663.

3 Ninomiya T, Kiyohara Y, Kubo M, Tanizaki Y, Doi Y, Okubo K, Wakugawa Y, Hata J, Oishi Y, Shikata K, Yonemoto K, Hirakata H, lida M. Chronic kidney disease and cardiovascular disease in a general Japanese population: the Hisayama Study. Kidney Int 2005; 68: 228-236.

4 Anavekar NS, McMurray JJ, Velazquez EJ, Solomon SD, Kober L, Rouleau JL, White HD, Nordlander R, Maggioni A, Dickstein K, Zelenkofske S, Leimberger JD, Califf RM, Pfeffer MA. Relation between renal dysfunction and cardiovascular outcomes after myocardial infarction. N Engl J Med 2004; 351: 1285-1295.

5 Zoungas S, Cameron JD, Kerr PG, Wolfe R, Muske C, McNeil JJ, McGrath BP. Association of carotid intima-medial thickness and indices of arterial stiffness with cardiovascular disease outcomes in CKD. Am J Kidney Dis 2007; 50: 622-630.

6 Shoji T, Emoto M, Shinohara K, Kakiya R, Tsujimoto Y, Kishimoto H, Ishimura E, Tabata T, Nishizawa Y. Diabetes mellitus, aortic stiffness, and cardiovascular mortality in endstage renal disease. J Am Soc Nephrol 2001; 12: 2117-2124.

7 Blacher J, Asmar R, Djane S, London GM, Safar ME. Aortic pulse wave velocity as a marker of cardiovascular risk in hypertensive patients. Hypertension 1999; 33: 1111-1117.

8 Terai M, Ohishi M, Ito N, Takagi T, Tatara Y, Kaibe M, Komai N, Rakugi H, Ogihara T. Comparison of arterial functional evaluations as a predictor of cardiovascular events in hypertensive patients: the Non-Invasive Atherosclerotic Evaluation in Hypertension (NOAH) study. Hypertens Res 2008; 31: 1135-1145.

9 Levey AS, Eckardt KU, Tsukamoto Y, Levin A, Coresh J, Rossert J, De Zeeuw D, Hostetter TH, Lameire N, Eknoyan G. Definition and classification of chronic kidney disease: a position statement from Kidney Disease: Improving Global Outcomes (KDIGO). Kidney Int 2005; 67: 2089-2100.

10 Imai E, Horio M, Nitta K, Yamagata K, Iseki K, Tsukamoto Y, Ito S, Makino H, Hishida A, Matsuo S. Modification of the Modification of Diet in Renal Disease (MDRD) Study equation for Japan. Am J Kidney Dis 2007; 50: 927-937.

11 Komai N, Ohishi M, Morishita R, Moriguchi A, Kaibe M, Matsumoto K, , Rakugi H, Higaki J, Ogihara T. Serum hepatocyte growth factor concentration is correlated with the forearm vasodilator response in hypertensive patients. Am J Hypertens 2002; 15: 499-506.

12 Nakagawa N, Takahashi F, Chinda J, Kobayashi M, Hayashi Y, Abe M, Saijo Y, Kikuchi K, Hasebe N. A newly estimated glomerular filtration rate is independently associated with arterial stiffness in Japanese patients. Hypertens Res 2008; 31: 193-201.

13 Tomiyama H, Tanaka H, Hashimoto H, Matsumoto C, Odaira M, Yamada J, Yoshida M, Shiina K, Nagata M, Yamashina A. Arterial stiffness and declines in individuals with normal renal function/early chronic kidney disease. Atherosclerosis 2010; 212: 345-350.

14 Mitchell GF, Hwang SJ, Vasan RS, Larson MG, Pencina MJ, Hamburg NM, Vita JA, Levy D, Benjamin EJ. Arterial stiffness and cardiovascular events: the Framingham Heart Study. Circulation 2010; 121: 505-511.

15 Mattace-Raso FU, van der Cammen TJ, Hofman A, van Popele NM, Bos ML, Schalekamp MA, Asmar R, Reneman RS, Hoeks AP, Breteler MM, Witteman JC. Arterial stiffness and risk of coronary heart disease and stroke: the Rotterdam Study. Circulation 2006; 113: 657-663.

16 Boutouyrie P, Tropeano Al, Asmar R, Gautier I, Benetos A, Lacolley P, Laurent S. Aortic stiffness is an independent predictor of primary coronary events in hypertensive patients: a longitudinal study. Hypertension 2002; 39: 10-15.

17 Libby P. Inflammation in atherosclerosis. Nature 2002; 450: 868-874.

18 Fearon IM, Faux SP. Oxidative stress and cardiovascular disease: novel tools give (free) radical insight. J Mol Cell Cardiol 2009; 47: 372-381.

19 Hage FG, Venkataraman R, Zoghbi GJ, Perry GJ, DeMattos AM, Iskandrian AE. The scope of coronary heart disease in patients with chronic kidney disease. J Am Coll Cardiol 2009; 53: 2129-2140.

20 Vlassara H, Torreggiani M, Post JB, Zheng F, Uribarri J, Striker GE. Role of oxidants/ inflammation in declining renal function in chronic kidney disease and normal aging. Kidney Int Supp/ 2009; 114: S3-S11. 
21 Tomiyama H, Arai T, Koji Y, Yambe M, Motobe K, Zaydun G, Yamamoto Y, Hori S, Yamashina A. The age-related increase in arterial stiffness is augmented in phases according to the severity of hypertension. Hypertens Res 2004; 27: 465-470.

22 Najjar SS, Scuteri A, Shetty V, Wright JG, Muller DC, Fleg JL, Spurgeon HP, Ferrucci L, Lakatta EG. Pulse wave velocity is an independent predictor of the longitudinal increase in systolic blood pressure and of incident hypertension in the Baltimore Longitudinal Study of Aging. J Am Coll Cardiol 2008; 51: 1377-1383.
23 Munakata M, Nagasaki A, Nunokawa T, Sakuma T, Kato H, Yoshinaga K, Toyota T. Effects of valsartan and nifedipine coat-core on systemic arterial stiffness in hypertensive patients. Am J Hypertens 2004; 17 (11 Part 1): 1050-1055.

24 Ichihara A, Hayashi M, Ryuzaki M, Handa M, Furukawa T, Saruta T. Fluvastatin prevents development of arterial stiffness in haemodialysis patients with type 2 diabetes mellitus. Nephrol Dial Transplant 2002; 17 : 1513-1517. 